\title{
Evidence of Kittel type behaviour of the permittivity of a nanostructured high sensitivity piezoelectric
}

Cite as: J. Appl. Phys. 123, 204103 (2018); https://doi.org/10.1063/1.5006011

Submitted: 21 September 2017 . Accepted: 08 May 2018. Published Online: 23 May 2018

Sergejus Balčiūnas, Maksim Ivanov (D), Robertas Grigalaitis, Juras Banys (D), Harvey Amorín (D), Alicia Castro, and Miguel Algueró

\section{ARTICLES YOU MAY BE INTERESTED IN}

Structural stability, enhanced magnetic, piezoelectric, and transport properties in (1-x)BiFeO $3^{-}$ (x)Bao.70Sro.30TiOz nanoparticles

Journal of Applied Physics 123, 204102 (2018); https://doi.org/10.1063/1.5023682

Role of $\mathrm{A}$-site $\mathrm{Ca}$ and $\mathrm{B}$-site $\mathrm{Zr}$ substitution in $\mathrm{BaTiO}_{3}$ lead-free compounds: Combined experimental and first principles density functional theoretical studies

Journal of Applied Physics 123, 204104 (2018); https://doi.org/10.1063/1.5021249

Flexoelectricity in antiferroelectrics

Applied Physics Letters 113, 132903 (2018); https://doi.org/10.1063/1.5044724

\section{AlluXa YOUROPTCAR}




\title{
Evidence of Kittel type behaviour of the permittivity of a nanostructured high sensitivity piezoelectric
}

\author{
Sergejus Balčiūnas, ${ }^{1}$ Maksim Ivanov, ${ }^{1, a)}$ Robertas Grigalaitis, ${ }^{1}$ Juras Banys, ${ }^{1}$ \\ Harvey Amorín, ${ }^{2}$ Alicia Castro, ${ }^{2}$ and Miguel Algueró ${ }^{2}$ \\ ${ }^{1}$ Faculty of Physics, Vilnius University, Sauletekio 9/3 817k., LT10222 Vilnius, Lithuania \\ ${ }^{2}$ Instituto de Ciencia de Materiales de Madrid, CSIC, Cantoblanco, 28049 Madrid, Spain
}

(Received 21 September 2017; accepted 8 May 2018; published online 23 May 2018)

\begin{abstract}
The broadband dielectric properties of high sensitivity piezoelectric $0.36 \mathrm{BiScO}_{3}-0.64 \mathrm{PbTiO}_{3}$ ceramics with average grain sizes from $1.6 \mu \mathrm{m}$ down to $26 \mathrm{~nm}$ were investigated in the $100-500 \mathrm{~K}$ temperature range. The grain size dependence of the dielectric permittivity was analysed within the effective medium approximation. It was found that the generalised core-shell (or brick wall) model correctly explains the size dependence down to the nanoscale. For the first time, the grain bulk and boundary properties were obtained without making any assumptions of values of the parameters or simplifications. Two contributions to dielectric permittivity of the grain bulk are described. The first is the size-independent one, which follows the Curie-Weiss law. The second one is shown to plausibly follow the Kittel's law. This seems to suggest the unexpected persistence of mobile ferroelectric domains at the nanoscale (26 nm grains). Alternative explanations are discussed. Published by AIP Publishing. https://doi.org/10.1063/1.5006011
\end{abstract}

\section{INTRODUCTION}

Ferroelectric and piezoelectric materials are highly attractive as they can be used in a variety of devices and there are still a number of fundamental issues that need to be understood. A highly topical problem is what happens to the properties of materials when some characteristic size is diminished down to the nanoscale. The studies cover everything from theory to basic research and applications. ${ }^{1-4}$

To rationalise the size dependence of the dielectric properties, one usually makes use of the effective medium approximation (EMA), which assumes the material irregularities to be much smaller than the wavelength of electromagnetic radiation. This allows the proposal and analysis of different models under the hypothesis of constant electric and magnetic fields inside each discrete region.

The most common models applied for ceramics are brick-wall (or brick-layer) and core-shell models, ${ }^{5,6}$ which converge to the same result when the volume fraction of the layer between the bricks or of the shell is small. ${ }^{6,7}$ In both cases, it is assumed that the grains of ceramics have the same properties as single crystals, and that the space between grains is filled with some different phase (inter-grain medium, or modified grain boundary), which has different dielectric properties. In the case of ferroelectric materials, this usually means that grain boundaries have a much lower permittivity than the bulk material. There have been attempts to apply this model to approximate the experimental dependence of dielectric properties on grain size. These studies provide values for the thickness and permittivity of the boundaries. It was, for example, found that the shell has a thickness of around $1-10 \mathrm{~nm}$ with a dielectric permittivity of 20-300 in the case of the canonical relaxor $\mathrm{PbMg}_{1 / 3} \mathrm{Nb}_{2 / 3} \mathrm{O}_{3}$

\footnotetext{
a) Author to whom correspondence should be addressed: maksim.ivanov@ ff.vu.lt
}

(PMN, bulk permittivity around 18000 at room temperature $)^{8}$ and $0.5-2 \mathrm{~nm}$ thickness with a permittivity of 50-200 in the case of conventional ferroelectric $\mathrm{BaTiO}_{3}$ (BTO, bulk permittivity around 2000 at room temperature). ${ }^{9,10}$ There definitely are more examples of application of EMA to describe the dielectric properties of ferroelectric ceramics, but not so many in the case of relaxor materials. Indeed, it seems that EMA cannot be directly applied in the latter case unless the size dependence of the core material properties is taken into account. ${ }^{8,11}$ A rather detailed review of the grain size effect on the dielectric properties of high-permittivity ceramics can be found in Ref. 10. As a short summary of the latter review, it must be noted, that in all cases, either the brick-wall model was used and the ratio of dielectric permittivity of the inter-grain material to the thickness of the inter-grain region was found, or the dielectric properties were analysed in accordance with the procedure proposed in Ref. 12. The latter allows to find both the dielectric properties and thickness of the inter-grain medium. However, these properties are assumed to be temperature independent, and the analysis is only possible in the paraelectric phase. This means that in all cases, some assumptions regarding properties of the inter-grain medium must be made to estimate either its thickness or dielectric permittivity.

Apart from dielectric studies, evidences of the existence of a grain boundary with a differentiated crystal structure were presented by Hoshina et al. ${ }^{13}$ Their synchrotron XRD study on BTO ceramics clearly indicated the existence of a cubic shell on the surface of the grain, whose thickness was found to be nearly independent of the grain size and close to $10-15 \mathrm{~nm}$. The difference between this value and those estimated from the dielectric properties could be due to the different techniques used and assumptions made.

We present here a comprehensive description of the dielectric properties of a high sensitivity piezoelectric 
$0.36 \mathrm{BiScO}_{3}-0.64 \mathrm{PbTiO}_{3}(0.36 \mathrm{BS}-0.64 \mathrm{PT})$ in the form of high density $(>95 \%)$ ceramics with average grain sizes of $1.6 \mu \mathrm{m}, 200 \mathrm{~nm}, 50 \mathrm{~nm}$, and $26 \mathrm{~nm}$. This composition corresponds to the morphotropic phase boundary of the material that has the temperature of ferroelectric to paraelectric phase transition at around $450{ }^{\circ} \mathrm{C} .{ }^{14}$ The results clearly indicate the presence of grain boundaries with differentiated properties, and of domain-like contribution to the permittivity of the grain bulk down to sizes as small as $26 \mathrm{~nm}$. The latter seem to be the effect of polar nano regions or ferroelectric nano domains, rather than conventional ferroelectric domains.

\section{EXPERIMENTAL}

Coarse grained ceramics were processed by conventional sintering of nanocrystalline powders obtained by mechanosynthesis, while submicron range and nanostructured ceramics were obtained by spark plasma sintering of the same powder under tailored conditions. Additional details can be found elsewhere. ${ }^{15,16}$ The distribution of grain sizes can be found in the supplementary material.

Dielectric measurements were performed in the $100-500 \mathrm{~K}$ temperature and $100 \mathrm{~Hz}-40 \mathrm{GHz}$ frequency ranges. A LCR-meter HP4284 was used to measure capacitance and loss tangent of the samples in the $100 \mathrm{~Hz}-1 \mathrm{MHz}$ frequency window. A coaxial line terminated with a capacitor was used in the $10 \mathrm{MHz}-1 \mathrm{GHz}$ band, in which measurements of complex reflection coefficient were performed with an Agilent 8714ET network analyser. Dielectric rods in waveguides were utilised in the $8-40 \mathrm{GHz}$ frequency range. The scalar reflection and transmission coefficients were measured in this case using a scalar network analyser Elmika R2400. Measurements were performed during the cooling cycle at a rate of $1 \mathrm{~K} / \mathrm{min}$. All sample contacts were made using silver paste.

\section{RESULTS AND DISCUSSION}

Temperature dependences of dielectric properties of ceramics with decreasing grain size down to the nanoscale are presented in Fig. 1. Here we can see that in the case of coarse grained ceramics, the dielectric losses do not show any pronounced maximum, at least not in our temperature range. However, a maximum is clearly present when the grain size is decreased, and dielectric losses look very similar to what would be expected from typical relaxors such as PMN. ${ }^{17-19}$ It must be noted, that coarse-grained ceramics of BS-PT with the same composition were also studied in Ref. 20. There, the relaxor-like anomaly at low temperatures was also observed, both in coarse grained ceramics and single crystals. The relaxor-like character was additionally confirmed in Ref. 21, where clear Vogel-Fulcher behaviour was reported. The origin of the anomaly was not analysed in detail, but it could be either polar nano regions or domain walls, as both can exhibit freezing behaviour. Similar anomalies had been also reported in other cases (i.e., Refs. 22 and 23) and they receive a lot of different explanations, which are beyond the scope of this paper (i.e., Refs. 24-27) However, in our case, the low temperature relaxation is clearly visible in submicron ceramics, while in the coarse

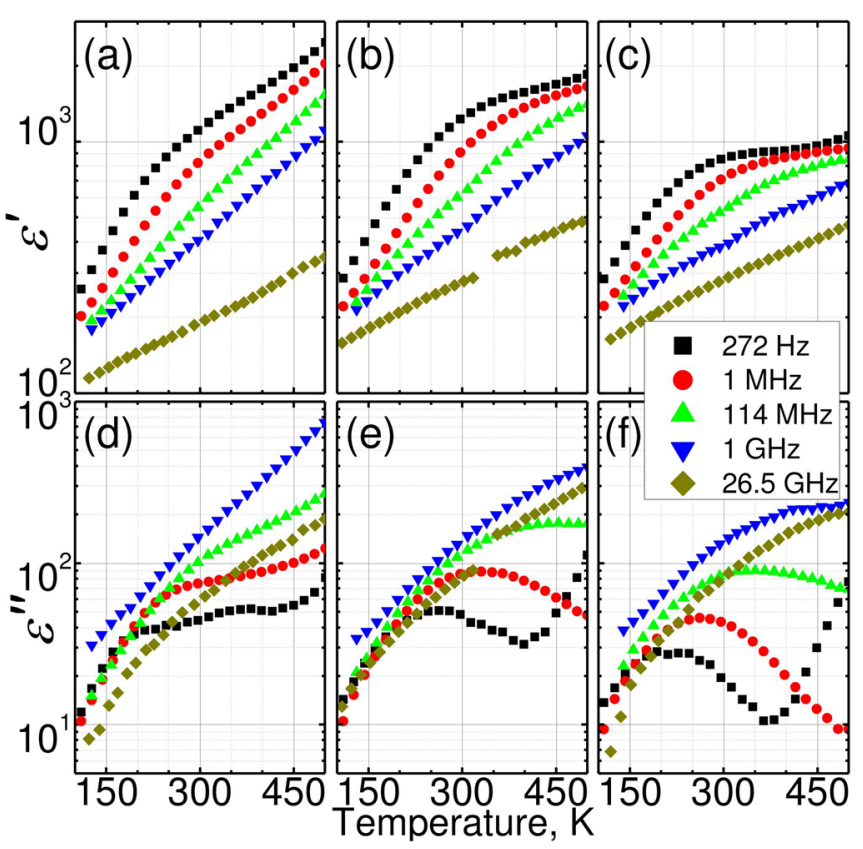

FIG. 1. Temperature dependences of the real $[(a)-(c)]$ and imaginary [(d)-(f)] parts of dielectric permittivity of ceramics with mean grain size $1.6 \mu \mathrm{m}[(\mathrm{a})$ and (d)], $50 \mathrm{~nm}[(\mathrm{~b})$ and (e)], and $26 \mathrm{~nm}$ [(c) and (f)].

grain case, the anomaly is somewhat smeared. The difference must stem from different processing techniques. The ceramics studied in Ref. 20 were produced using conventional (mixed oxide) means, while in our case, the starting powder was made using mechanosynthesis. This means that a significant crystalline strain is introduced in our case, ${ }^{16}$ which might be the reason for the differences in the case of coarse-grained ceramics. In the case of smaller grains, the crystalline strain is still there, but the grain boundary dilution both diminishes the real part of dielectric permittivity and makes the relaxor-like process narrower in the temperature domain. Some additional insights to the dynamic properties of these ceramics are presented elsewhere. ${ }^{21}$

The data presented in Fig. 1 was given a deeper analysis from the perspective of the grain size effect. In this work, we decided to apply the generalised core-shell model (also known as the generalised brick-wall model), ${ }^{7}$ which is valid for densely packed cores of any shape separated by thin shells, to recover intrinsic properties of the ceramics: thickness and dielectric permittivity of the shell and properties of the bulk material. The relation between intrinsic properties and effective (experimentally determined) dielectric permittivity is as follows:

$$
\varepsilon=\varepsilon_{S} \frac{x-n}{1-n}+\frac{1-x}{1-n} \cdot \frac{\varepsilon_{C} \varepsilon_{S}}{(1-n) \varepsilon_{S}+n \varepsilon_{C}} .
$$

Here, $\varepsilon_{S}$ is the dielectric permittivity of the shell material, $\varepsilon_{C}$ is the dielectric permittivity of the bulk (core) material, $x$ is the volume fraction of the shell, and parameter $n=x / 3$ takes into account topology of the composite $(0-3$ connectivity). The depolarisation factor of $1 / 3$ corresponds to the spherical symmetry of grains. In the general case, the factor must be between 0 and 1 . However, in our case, the grains are nearly equiaxed polyhedra. The crystal itself 
should not be expected to have a great anisotropy, especially due to the polydomain state. In such cases, the depolarisation factor of $1 / 3$ is shown to be a quite good approximation. ${ }^{5,10}$

If the shell thickness is assumed to be independent of the grain size, then the volume fraction of the shell can be calculated as follows:

$$
x=1-\left(\frac{D-2 \cdot d}{D}\right)^{3} .
$$

Here, $D$ is the diameter of the grain and $d$ is the thickness of the shell.

However, our material is a ferroelectric, thus one must take domain wall contribution to the total dielectric permittivity of the grain bulk into account. ${ }^{28-30}$ It should be proportional to the concentration of domain walls. It is known from literature, that domains are expected to be plate-like in ferroelectric ceramics, with the domain size following Kittel's law. ${ }^{1,2,31}$ Thus, we take into account size dependence of dielectric permittivity of the bulk material

$$
\varepsilon_{C}=\varepsilon_{C 0}+\frac{A}{\sqrt{D}} .
$$

Here, $\varepsilon_{C 0}$ is the grain size-independent dielectric permittivity, and $A$ is a proportionality parameter for contribution of domain walls. An exemplary approximation is presented in Fig. 2 [see the black solid line in (a) and (b)]. Alternatively, we tried to approximate the data by a simpler function without any size dependence of the permittivity of the bulk medium. This does not allow to describe the maximum in permittivity, so we had to omit the ceramics with $1.6 \mu \mathrm{m}$ grain size [see the red dashed line in Figs. 2(a) and

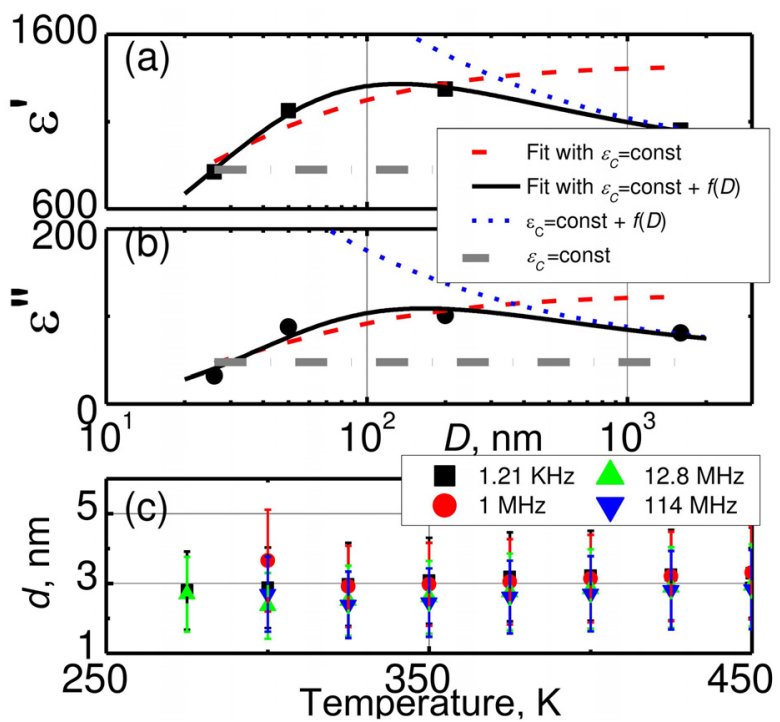

FIG. 2. Grain size dependence of the real (a) and imaginary (b) parts of dielectric permittivity of ceramics at $1 \mathrm{MHz}$ at room temperature (red dashed line-approximation of the size dependence without Kittel-type behaviour, solid black line-approximation with Kittel-type behaviour, blue shortdashed line-dielectric permittivity of the bulk with Kittel-type contribution, and grey dashed line-grain size-independent dielectric permittivity of the bulk); (c) temperature dependence of shell thickness at various frequencies. 2(b)]. As we can see, the approximation including the domain wall contribution is much better, it can include all the available experimental points and thus explain the presence of a maximum. A similar idea was previously used by others (e.g., Refs. 32 and 33) but it was usually applied only to describe the increase in dielectric permittivity upon grain size reduction without taking the grain boundary dilution into account. Furthermore, in our case, no restrictions were made during the optimisation process to obtain the fit curve, as we have 8 experimental points (both real and imaginary parts included), and there are 7 mathematically unrelated parameters to optimise (real and imaginary parts of $\varepsilon_{S}, \varepsilon_{C 0}$, and $A$ and thickness of the shell $d$ ). However, due to effective series electrical connection of the grain bulk and the shell, a strong statistical interdependence of the parameters is observed. Normally, this problem would be solved by fixing the shell thickness, e.g., Refs. 9, 10, 12, and 34. However, in our case, only permittivity of the shell is rescaled, while other parameters remain unchanged within their respective uncertainty interval. Therefore, we chose to optimize $\varepsilon_{S}$ and $d$ independently.

The values of dielectric permittivity of the shell $\varepsilon_{S}$ and its thickness $d$ discussed further on are the ones, which minimise the $\chi^{2}$ (sum of the squared deviations of the theoretical value from the experimental points) value of the approximation. One of the best ways to validate the result is to check if the temperature dependences of its parameters are reasonable. Figure 2(c) contains temperature dependence of shell thickness at various frequencies. The error bars are overestimated to show the significance of interdependence. Furthermore, the error actually represents a scaling coefficient, which is the same for all values. The shape of the dependence would remain the same. As we can see, the thickness is nearly constant and is independent of the experiment (frequencies of $1.21 \mathrm{kHz}$ and $1 \mathrm{MHz}$ are one measurement, and $12.8 \mathrm{MHz}$ and $114 \mathrm{MHz}$ are from a totally different one, performed on different samples and using a different technique). This is expected in the case of a perfect core-shell structure, and the $d$ is in the range of $2.5-3.5 \mathrm{~nm}$. This means that the total thickness of the intergrain material is $5-7 \mathrm{~nm}$. It must be noted, that this thickness does not necessarily represent the thickness of some second phase. It is possible, that the intergrain material is actually chemically and structurally the same, as the bulk of the grain. However, the ferroelectric domains could be clamped to the surface, so this volume of the grain would have smaller dielectric response. A similar idea was previously proposed for the case of PMN by Papet et al..$^{35}$ and for BTO by Polotai et al. ${ }^{36}$

Perhaps, a few words must be said about the frequency and temperature interval selection. The approximations are only reasonable if there is a substantial size dependence of the permittivity. This is only visible at relatively small frequencies (below $1 \mathrm{GHz}$ ) and in a limited temperature range (above 250-300 K). The lower limit is set by the decrease in permittivity (see Fig. 1), where grain boundary dilution becomes of minor effect. The upper limit of $450 \mathrm{~K}$ is set by conductivity effects, which seem to be dependent on defects, introduced into the system, and their behaviour is dependent 
on the sample (why-is a question for a different investigation).

A major issue of the approach taken is the use of Kittel's law. First of all, perhaps a different power than $1 / 2$ might be relevant for the scaling in Eq. (3). The available experimental data allows to conclude, the contribution of domain walls can scale as $A / D^{n}$, where $0.3 \leq n \leq 1.3$ (see supplementary material). The smallest $\chi^{2}$ value was obtained with $n=0.77$. So, is the law square-root or linear? We cannot distinguish. But we can rule out higher and smaller powers. The square-root is physically justified and has some (albeit limited) confirmation in literature, ${ }^{2,37,38}$ so it seems to be the best option. Thus, further analysis assumes validity of Eq. (3).

The second major issue is the use of Kittel's law for nanostructured ceramics with a grain size of $26 \mathrm{~nm}$, and the assumption that multidomain grains exist for such small grain sizes. Processing of ceramics is carried out above the Curie temperature and thus, at the cubic phase. During the cooling through the phase transition, the deformation of the individual grains is restricted by the surroundings. This way, ferroelectric/ferroelastic domains (i.e., $90^{\circ}$ ) are formed. However, at very small grains, the density of ferroelastic domains becomes too large to be energetically favourable. As a result, monodomain grains are favoured in the case of small particles inside the rigid matrix. However, this does not take into account the inversion domains $\left(180^{\circ}\right)$. Their formation must be governed by the depolarising fields at the grain boundaries. Besides, in the case of ceramics, some mobile (even if very slow) charges may be present, especially at the grain boundaries. It was experimentally observed that grains are single domain when they are smaller than $100 \mathrm{~nm},{ }^{1,39}$ so the proposed model is not expected to hold in nanograined ceramics. However, there might be some other entities contributing to dielectric permittivity, i.e., polar nano regions. Actually, the material does show low temperature relaxor-like behaviour. It is also possible that there are structural nanotwins, which are expected to be present in a ferroelectric composition close to a morphotropic phase boundary. ${ }^{40-42}$ It is known that the twins can change their size under the electric field and mechanical strain (e.g., Refs. 40 and 42) so they can give a contribution to dielectric permittivity and could be interpreted as ferroelectric nano domains. Furthermore, it was recently showed, that the polar nano regions coexisting within the ferroelectric domains can contribute substantially to the dielectric permittivity and piezoelectricity in the case of morphotropic relaxor-PT solid solutions. ${ }^{43}$ It is worth remarking that previous studies reporting monodomain grains used piezoresponse force microscopy (PFM), and that technique resolution is limited by the size of the probe tip $(\sim 20 \mathrm{~nm})$. Therefore, smaller domain erntities cannot be resolved. ${ }^{44}$ However, ferroelectric switching was confirmed even in the nanoscale grains. Similar results have been observed in coarse grained ceramics of a related solid solution BS-BT $\left(\mathrm{BiScO}_{3}-\mathrm{BaTiO}_{3}\right)$, but explained by polar nanoregions alone as local ferroelectricity was confirmed by PFM. ${ }^{45}$ Another reason to believe the polar entities following the Kittel's law are not the conventional domains is the fact that, based on the theoretical approximation, the permittivity would be maximum in ceramics with approximately $200 \mathrm{~nm}$ grain size. In comparison, the permittivity is maximum at around $1 \mu \mathrm{m}$ grain size in conventional ferroelectric BTO. ${ }^{9}$ Furthermore, the thickness of the intergrain material obtained from the analysis is quite large. The intergrain medium could be thick if it was a region of the grain with clamped polar entities. The medium would be allowed to have a relatively high permittivity of hundreds in this case. Nevertheless, and whatever the mechanism is, the Kittel's law seems to hold at the nanoscale.

Another interesting point is that the contribution of the size-dependent process is quite large, exactly as it was shown for morphotropic relaxor-PT solid solutions, ${ }^{43}$ and it can be much higher than the contribution of either phonons or conventional ferroelectric domains. The blue short-dashed line in Figs. 2(a) and 2(b) corresponds to formula (3). We can see that the size-dependent contribution is around 20\% to the real part of permittivity value of the grain core in the case of coarse-grained ceramics, and reaches nearly $50 \%$ in submicron grains (compare with the grey dashed line, representing grain size-independent contribution).

Figure 3 shows all other fitting parameters, which allow further conclusions to be reached. First, the size-independent part of dielectric permittivity of the core [see Figs. 3(a) and 3(b)] exhibits quite broad dipolar relaxation. The mean relaxation time is bound to correspond to frequencies higher than $100 \mathrm{MHz}$, as dielectric losses increase with frequency. It is also important to note that this contribution might partially incorporate contribution of domain walls, which should be present in coarse-grained ceramics. This incorporation can be the source of fitting errors. However, the temperature dependence of the size-independent part of dielectric permittivity follows the Curie-Weiss law (see supplementary material). It means, that this contribution is, at least partially, due to the phonons. However, according to Ref. 20, the phonon contribution is not expected to exceed the value of 100-120. The other contribution is likely related to electromechanical coupling and shear wave generation. ${ }^{20,29,33}$ However, this contribution is inversely proportional to $P_{0}^{2}$, that is $\Delta \varepsilon$ $\sim P_{0}{ }^{-2} \sim\left(T-T_{C}\right)^{-1}$. In other words, this contribution follows the Curie-Weiss law in the case of weak variation of mechanical properties with temperature, and is indistinguishable from phonon contribution in this regard.

Second, we can analyse the temperature dependence of the size-dependent contribution [Figs. 3(e) and 3(f)]. This contribution is a priori expected to be frequency dependent, as in the case of normal ferroelectrics, the domain wall dynamics show relaxation somewhere in the $1 \mathrm{~Hz}-1 \mathrm{MHz}$ range, depending on the temperature and material (e.g., Refs. 28,30 , and 46) In our case, dielectric losses clearly show a frequency-dependent maximum. At low temperatures the losses become frequency-independent. This is quite similar to the behaviour of ferroelectric relaxor materials. The same property is observable at lower temperatures in the ceramics themselves (Fig. 1). The shift of the temperature of the anomaly in the case of ceramics can be explained in the frame of EMA. The size-independent permittivity increases upon heating, while size-dependent contribution seems to saturate. Grain boundary dilution leads to the shift of the 

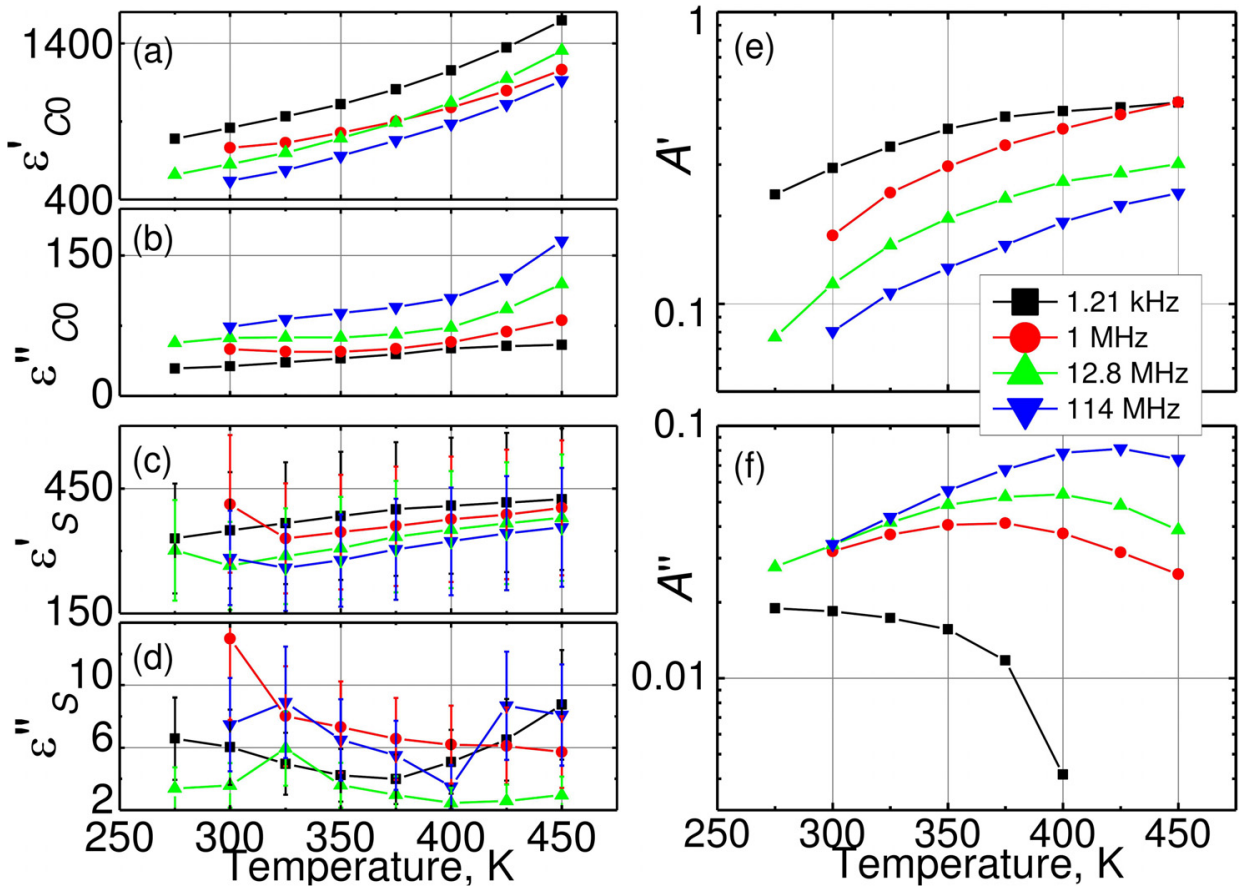

FIG. 3. Temperature dependences of the real and imaginary parts of grain size independent dielectric permittivity of the bulk material [(a) and (b)], of the shell material [(c) and (d)], and real and imaginary coefficients of contribution of grain size dependent contribution [(e) and (f)]. mean relaxation time to higher frequencies. ${ }^{6,7,21}$ This would lead to an apparent temperature shift of the anomaly related to the size-dependent process towards lower temperatures.

We can see that at high temperatures losses of the grain size-dependent process are very close to 0 at frequencies below $1 \mathrm{MHz}$, and size-dependent permittivity has the same value at $1 \mathrm{kHz}$ and $1 \mathrm{MHz}$ at $450 \mathrm{~K}$; but it is different at lower temperatures. This means that the relaxation time shortens upon heating. The width of the distribution of the relaxation times must be finite at $450 \mathrm{~K}$ temperature. Frequency-independent losses at low temperatures mean, on the other hand, an infinitely broad dispersion region. This means that the process becomes wider upon cooling. This is the same behaviour as in the case of ferroelectric relaxors, ${ }^{22,23}$ and is usually attributed to the polar nano regions. This is, again, in favour of the same explanation provided in Ref. 43. However, this model does not provide any explanation for the Kittel-type behaviour. Thus, ferroelectric nanodomains, e.g., as proposed in Ref. 47, seem to be a better candidate.

Third, the dielectric permittivity of the shell material [see Figs. 3(c) and 3(d)] is not as small as it was in the case of BTO: $250-450$ compared to $100 .^{9}$ As noted above, the value of the permittivity presented here is an estimate, and the error bars are of the same function as in the case of Fig. 2(c). If the true value of the shell thickness is somewhat different, then both the real and imaginary parts would be rescaled (however, not linearly proportionally to the change of the thickness). Still, all other features (relaxations, temperature dependence trends) would remain the same. It is also interesting to note, that the shell has quite small and frequency independent dielectric losses. However, the losses cannot be neglected, as the real part clearly shows frequency dependence. The dispersion is quite broad, and this suggests the grain boundary material to be disordered. This can explain the relatively large permittivity of the shell, which is clearly bigger than the pure phonon contribution. Possibly, some kind of dipoles, related to charge separation or some kind of polar defect (vacancy) complexes are contributing to the relatively large value. The permittivity value, however, is quite similar to effective dielectric properties at $26 \mathrm{GHz}$ of the ceramics (see Fig. 1). This would explain the lack of size dependence of the dielectric permittivity at high frequencies. If the shell material has the same properties as the bulk, then grain boundary dilution will not be observed. The same phenomenon happens at all frequencies at temperatures below $270 \mathrm{~K}$.

\section{SUMMARY}

In summary, we succeeded in applying the core-shell effective medium model to nanostructured ceramics of $0.36 \mathrm{BiScO}_{3}-0.64 \mathrm{PbTiO}_{3}$ and in differentiating the grain bulk and boundary properties without making any additional assumptions or simplifications. We found that a grain boundary with a total thickness of around $6 \mathrm{~nm}$ and a reduced permittivity is present. The dielectric properties of this shell are temperature and frequency dependent with a broad dispersion region, meaning the medium is quite disordered. Most likely, this region is very close in its constitution to the bulk material, but the polar entities inside it are clamped by the surface of the grain. The permittivity of the grain bulk requires considering 2 contributions: a size-independent one which is due to phonons and shear wave generation (electromechanical contribution) and follows the Curie-Weiss law, and a second contribution that depends on the grain size, which is shown to adequately follow the Kittel's law. The latter process gives an important contribution to the dielectric permittivity, and is the source of relaxor-like behaviour of the ceramics. Though the Kittel's law is usually related to conventional ferroelectric domains, it is hard to assume their existence in nanostructured materials. Thus, it might instead be 
related to the contribution of polar nanoregions (which are embedded within the ferroelectric, possibly multi-domain, matrix), or, alternatively, ferroelectric nanodomains.

\section{SUPPLEMENTARY MATERIAL}

See supplementary material for additional data and comments.

\section{ACKNOWLEDGMENTS}

This work was supported by project TUMOCS. This project has received funding from the European Union's Horizon 2020 research and innovation programme under the Marie Skłodowska-Curie [Grant Agreement No. 645660]. Financial support of the Spanish MINECO (Project Nos. MAT2014-58816-R and MAT2017-88788-R) and the collaboration in the frame of COST MP0904 Action are highly acknowledged.

${ }^{1}$ G. Arlt, J. Mater. Sci. 25, 2655 (1990).

${ }^{2}$ W. Cao and C. A. Randall, J. Phys. Chem. Solids 57, 1499 (1996).

${ }^{3}$ M. Dawber, K. M. Rabe, and J. F. Scott, Rev. Mod. Phys. 77, 1083 (2005).

${ }^{4}$ J. Banys, M. Ivanov, J. Macutkevic, A. Krotkus, H. J. Fan, S. Kawasaki, and J. F. Scott, Ferroelectrics 378, 79 (2009).

${ }^{5}$ N. J. Kidner, N. H. Perry, T. O. Mason, and E. J. Garboczi, J. Am. Ceram. Soc. 91, 1733 (2008)

${ }^{6}$ J. Petzelt, I. Rychetsky, and D. Nuzhnyy, Ferroelectrics 426, 171 (2012).

${ }^{7}$ I. Rychetsky and J. Petzelt, Ferroelectrics 303, 137 (2004).

${ }^{8}$ J. Carreaud, C. Bogicevic, B. Dkhil, and J. M. Kiat, Appl. Phys. Lett. 92, 242902 (2008).

${ }^{9}$ M. T. Buscaglia, M. Viviani, V. Buscaglia, L. Mitoseriu, A. Testino, P. Nanni, Z. Zhao, M. Nygren, C. Harnagea, D. Piazza, and C. Galassi, Phys. Rev. B 73, 064114 (2006).

${ }^{10} \mathrm{~J}$. Petzelt, Ferroelectrics 400, 117 (2010).

${ }^{11}$ R. Grigalaitis, M. Ivanov, J. Macutkevic, J. Banys, J. Carreaud, J. M. Kiat, V. V. Laguta, and B. Zalar, J. Phys. Condens. Matter 26, 272201 (2014).

${ }^{12}$ A. Y. Emelyanov, N. A. Pertsev, S. Hoffmann-Eifert, U. Böttger, and R. Waser, J. Electroceram. 9, 5 (2002).

${ }^{13}$ T. Hoshina, S. Wada, Y. Kuroiwa, and T. Tsurumi, Appl. Phys. Lett. 93, 192914 (2008).

${ }^{14}$ R. E. Eitel, C. A. Randall, T. R. Shrout, P. W. Rehrig, W. Hackenberger, and S.-E. Park, Jpn. J. Appl. Phys., Part 1 40, 5999 (2001).

${ }^{15}$ T. Hungría, H. Amorín, M. Algueró, and A. Castro, Scr. Mater. 64, 97 (2011).

${ }^{16}$ M. Algueró, P. Ramos, R. Jiménez, H. Amorín, E. Vila, and A. Castro, Acta Mater. 60, 1174 (2012).

${ }^{17}$ R. A. Cowley, S. N. Gvasaliya, S. G. Lushnikov, B. Roessli, and G. M. Rotaru, Adv. Phys. 60, 229 (2011).
${ }^{18}$ A. A. Bokov and Z.-G. Ye, Frontiers of Ferroelectricity (Springer US, 2007), pp. 31-52.

${ }^{19}$ A. A. Bokov and Z.-G. Ye, J. Adv. Dielectr. 02, 1241010 (2012).

${ }^{20}$ V. Porokhonskyy, S. Kamba, A. Pashkin, M. Savinov, J. Petzelt, R. E. Eitel, and C. A. Randall, Appl. Phys. Lett. 83, 1605 (2003).

${ }^{21}$ S. Balčiūnas, M. Ivanov, R. Grigalaitis, J. Banys, H. Amorín, A. Castro, and M. Algueró, Phys. Status Solidi B 252, 2727 (2015).

${ }^{22}$ S. Kamba, V. Bovtun, J. Petzelt, I. Rychetsky, R. Mizaras, A. Brilingas, J. Banys, J. Grigas, and M. Kosec, J. Phys. Condens. Matter 12, 497 (2000).

${ }^{23}$ J. Macutkevic, S. Kamba, J. Banys, A. Brilingas, A. Pashkin, J. Petzelt, K. Bormanis, and A. Sternberg, Phys. Rev. B 74, 104106 (2006).

${ }^{24}$ V. V. Kirillov and V. A. Isupov, Ferroelectrics 5, 3 (1973).

${ }^{25}$ O. Bidault, P. Goux, M. Kchikech, M. Belkaoumi, and M. Maglione, Phys. Rev. B 49, 7868 (1994).

${ }^{26}$ O. Bidault, M. Maglione, M. Actis, M. Kchikech, and B. Salce, Phys. Rev. B 52, 4191 (1995).

${ }^{27}$ A. K. Tagantsev, Phys. Rev. Lett. 72, 1100 (1994).

${ }^{28}$ W. Kleemann, Annu. Rev. Mater. Res. 37, 415 (2007).

${ }^{29}$ G. Arlt, U. Böttger, and S. Witte, Ann. Phys. 506, 578 (1994).

${ }^{30}$ B. L. Cheng, M. Gabbay, M. Maglione, Y. Jorand, and G. Fantozzi, J. Phys. IV 06, C8 (1996).

${ }^{31}$ D. Ghosh, A. Sakata, J. Carter, P. A. Thomas, H. Han, J. C. Nino, and J. L. Jones, Adv. Funct. Mater. 24, 885 (2014).

${ }^{32}$ G. Arlt, D. Hennings, and G. de With, J. Appl. Phys. 58, 1619 (1985).

${ }^{33}$ T. Hoshina, K. Takizawa, J. Li, T. Kasama, H. Kakemoto, and T. Tsurumi, Jpn. J. Appl. Phys., Part 147, 7607 (2008).

${ }^{34}$ M. H. Frey, Z. Xu, P. Han, and D. A. Payne, Ferroelectrics 206, 337 (1998).

${ }^{35}$ P. Papet, J. P. Dougherty, and T. R. Shrout, J. Mater. Res. 5, 2902 (1990).

${ }^{36}$ A. V. Polotai, A. V. Ragulya, and C. A. Randall, Ferroelectrics 288, 93 (2003).

${ }^{37}$ T. Hoshina, Y. Kigoshi, S. Hatta, T. Teranishi, H. Takeda, and T. Tsurumi, Ferroelectrics 402, 29 (2010).

${ }^{38}$ V. Porokhonskyy, L. Jin, and D. Damjanovic, Appl. Phys. Lett. 94, 212906 (2009).

${ }^{39}$ H. Amorín, R. Jiménez, J. Ricote, T. Hungría, A. Castro, and M. Algueró, J. Phys. D: Appl. Phys. 43, 285401 (2010).

${ }^{40}$ Y. Zhang, D. Xue, H. Wu, X. Ding, T. Lookman, and X. Ren, Acta Mater. 71, 176 (2014).

${ }^{41}$ Y. Huan, X. Wang, Z. Shen, J. Kim, H. Zhou, and L. Li, J. Am. Ceram. Soc. 97, 700 (2014)

${ }^{42}$ R. Theissmann, L. A. Schmitt, J. Kling, R. Schierholz, K. A. Schönau, H. Fuess, M. Knapp, H. Kungl, and M. J. Hoffmann, J. Appl. Phys. 102, 024111 (2007).

${ }^{43}$ F. Li, S. Zhang, T. Yang, Z. Xu, N. Zhang, G. Liu, J. Wang, J. Wang, Z. Cheng, Z.-G. Ye, J. Luo, T. R. Shrout, and L.-Q. Chen, Nat. Commun. 7, 13807 (2016).

${ }^{44}$ N. Salazar, M. Algueró, H. Amorín, A. Castro, A. Gil, and J. Ricote, J. Appl. Phys. 116, 124108 (2014).

${ }^{45}$ H. Y. Guo, C. Lei, and Z.-G. Ye, Appl. Phys. Lett. 92, 172901 (2008).

${ }^{46}$ P. Kubinec, M. Fally, A. Fuith, H. Kabelka, and C. Filipic, J. Phys. Condens. Matter 7, 2205 (1995).

${ }^{47}$ J. Hlinka, J. Adv. Dielectr. 02, 1241006 (2012). 